\title{
Capacitor-loaded split ring resonators as tunable metamaterial components
}

K. Aydin, and E. Ozbay

Citation: Journal of Applied Physics 101, 024911 (2007); doi: 10.1063/1.2427110

View online: http://dx.doi.org/10.1063/1.2427110

View Table of Contents: http://aip.scitation.org/toc/jap/101/2

Published by the American Institute of Physics

\section{Articles you may be interested in}

Electromagnetic resonances in individual and coupled split-ring resonators Journal of Applied Physics 92, 2929 (2002); 10.1063/1.1497452

Frequency tunable electromagnetic metamaterial using ferroelectric loaded split rings Journal of Applied Physics 103, 066105 (2008); 10.1063/1.2898575

Electric coupling to the magnetic resonance of split ring resonators Applied Physics Letters 84, 2943 (2004); 10.1063/1.1695439

Electric-field-coupled resonators for negative permittivity metamaterials Applied Physics Letters 88, 041109 (2006); 10.1063/1.2166681

Electrically small split ring resonator antennas Journal of Applied Physics 101, 083104 (2007); 10.1063/1.2722232

Split ring resonator sensors for infrared detection of single molecular monolayers Applied Physics Letters 95, 043113 (2009); 10.1063/1.3194154

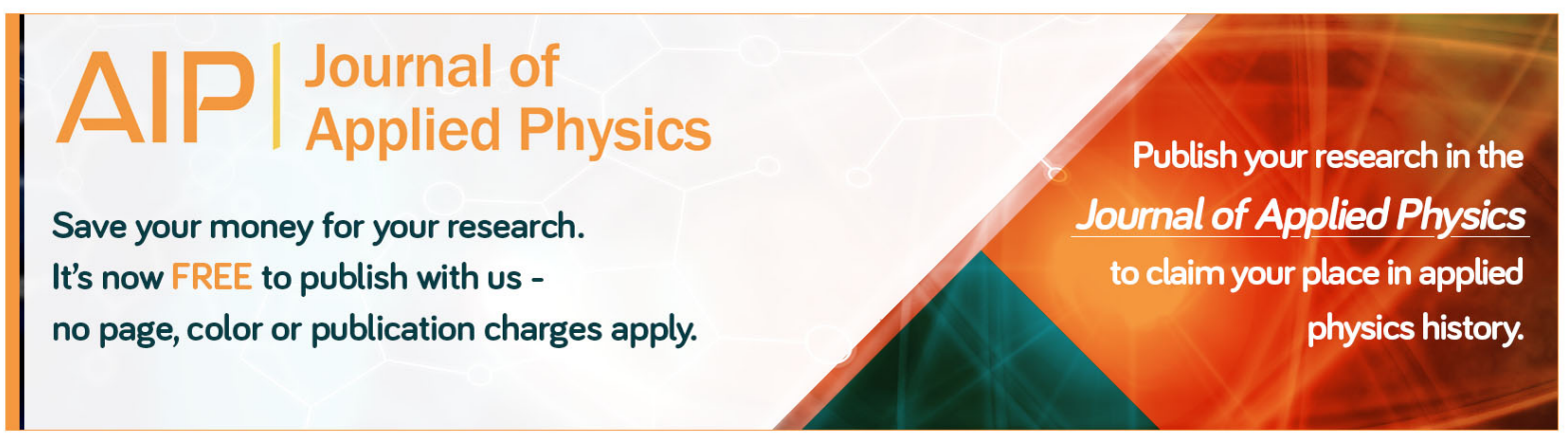




\title{
Capacitor-loaded split ring resonators as tunable metamaterial components
}

\author{
K. Aydin ${ }^{\text {a) }}$ and E. Ozbay \\ Nanotechnology Research Center, Bilkent University, Bilkent, Ankara 06800, Turkey; \\ Department of Physics, Bilkent University, Bilkent, Ankara 06800, Turkey; \\ and Department of Electrical and Electronics Engineering, Bilkent University, Bilkent, \\ Ankara 06800, Turkey
}

(Received 3 August 2006; accepted 9 November 2006; published online 22 January 2007)

\begin{abstract}
Transmission through split ring resonator (SRR) structures loaded with capacitors is investigated both experimentally and numerically. Magnetic resonance frequency $\left(\omega_{m}\right)$ is observed to shift to lower frequencies when capacitors are mounted to the various capacitive regions of the SRR structure. The amount of change in $\omega_{m}$ depends strongly on the place where the capacitors are loaded. The magnetic resonance is obtained at $0.99 \mathrm{GHz}$ with subwavelength SRR size of $\lambda / 42$ when the capacitor $(C=2.2 \mathrm{pF})$ is integrated at the split region of the outer ring. (C) 2007 American Institute of Physics. [DOI: 10.1063/1.2427110]
\end{abstract}

\section{INTRODUCTION}

Artificially constructed materials may have properties that are not available in naturally occurring materials. As such an exciting composite material, split ring resonators (SRRs) received growing interest in recent years. Exotic electromagnetic (EM) properties emerge from SRR structures such as negative permeability and left-handed (LH) electromagnetism. Pendry et al. verified that SRRs built from nonmagnetic thin sheets of metal possess a wide range of effective magnetic permeability values including the negative ones. ${ }^{1}$ The scale of SRR is much less than the incident EM radiation, so that the effective medium theory is applicable. It has been shown that SRR array, when combined with proper thin wire array, exhibits left-handed properties. ${ }^{2-5}$ Since SRRs play an important role for the construction of left-handed metamaterials (LHMs) with negative refractive index, considerable amount of effort has been given to understand the underlying physics of these negative permeability materials in the literature. ${ }^{6-14}$ Numerical simulations and analytical models are employed to calculate capacitance values and resonance frequencies of various SRR designs. $^{9-12}$

The LH passband frequency is essentially determined by the magnetic resonance frequency $\left(\omega_{m}\right)$ of periodic SRR structures. Controlling $\omega_{m}$ of SRRs will open ways for operation of negative refractive media at the desired range of frequencies. Changing the $\omega_{m}$ of SRR structure can be achieved by changing the capacitance or inductance of the split ring resonators. Recently Gil et al. loaded varactor diodes on SRR-based notch filters for tuning the frequency of operation. ${ }^{13}$ In this study, we present experimental results of transmission through the unit cell of SRRs loaded with capacitors at different capacitive regions.

\section{STRUCTURES AND EXPERIMENTAL SETUP}

Split ring resonator structures are built from nonmagnetic concentric copper rings with splits oriented at the op-

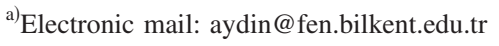

posite sides. Figure 1(a) shows a schematic drawing of a single SRR. The structural parameters, as provided in Fig. 1(a), are $d=t=0.2 \mathrm{~mm}, w=0.9 \mathrm{~mm}$, and $R=3.6 \mathrm{~mm}$. Planar SRR array is fabricated on FR4 printed circuit boards, and unit cells of SRRs are extracted from the array. Surface mount capacitors with various capacitance values are placed at three different capacitive regions of SRRs. Namely, (i) the gap region between inner and outer rings, (ii) outer ring's split region, and (iii) inner ring's split region. The photographs of the SRRs loaded with capacitors are given in the insets of Figs. 2(a)-2(c), respectively.

The resonance behavior could be observed from the frequency response of single SRR. Two monopole antennas are used to transmit and detect the EM waves through the single SRR unit cell. The monopole antenna was constructed by removing the shield around one end of a microwave coaxial cable. The exposed center conductor which also acted as the transmitter and receiver was on the order of $\lambda / 2$, arranged to work at the frequency range covering the $\omega_{m}$ of the SRR structures. A single SRR is placed between the monopole antennas as shown in Fig. 1(b). The distance between the monopole and SRR unit cell is $6 \mathrm{~mm}$. Monopole antennas are then connected to the HP-8510C network analyzer to measure the transmission coefficients. First, we measured the transmission spectra in free space (i.e., without SRR unit cell), which is used as the calibration data for the network analyzer. Then, SRR unit cell was inserted between the monopole antennas, and we performed the transmission measurements by maintaining the distance between the transmitter and receiver monopole antennas fixed.
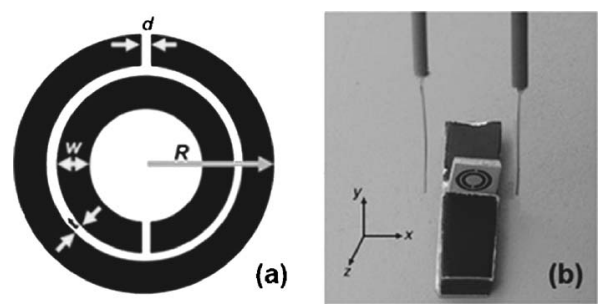

FIG. 1. (a) Schematic picture of a single split ring resonator. (b) Photograph of experimental setup for measuring transmission coefficients. 


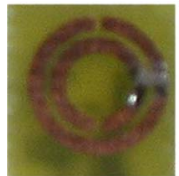

(a)

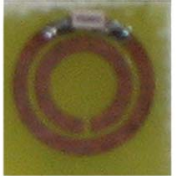

(b)

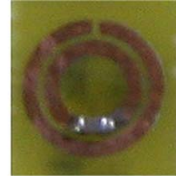

(c)
FIG. 2. (Color online) SRR with capacitor loaded (a) in the gap region between concentric rings, (b) in the outer split region, and (c) in the inner split region.

\section{RESULTS}

Split ring resonators consist of two concentric rings separated by a gap, both having splits oriented at opposite sides. Magnetic resonance is induced by the splits at the rings and the gap between the concentric rings. Current cannot flow around any one ring because of the splits, but the capacitance at the gap between the two rings enables current to flow. ${ }^{1}$ SRRs can be modeled as $L C$ circuits where the inductance arises from the rings. ${ }^{9,10}$ Total capacitance of the SRR system has mainly two contributions. The first one is the capacitance at the split regions and the second contribution is from the gap between the concentric rings. These capacitances together with the inductances from the rings determine the $\omega_{m}$ of the resonator structure. Changing the capacitance and inductance values results in a change in $\omega_{m}$ of SRRs, as expected from an $L C$ circuit. Recently Aydin et al. studied the effects of several SRR parameters such as split width, gap distance, and metal width on magnetic resonance frequency of SRRs. ${ }^{6}$

Magnetic resonance frequency of SRR without any additional capacitance is measured at $3.82 \mathrm{GHz}$ (data not shown here). ${ }^{5}$ Firstly, we mounted capacitors at the gap region between the inner and outer rings [see Fig. 2(a)]. The capacitance at the gap region is therefore changed, which affects the total capacitance of the system. Measured transmission coefficients of SRRs loaded with different capacitances are provided in Fig. 3(a). The magnetic resonance frequency is reduced down to $3.44 \mathrm{GHz}$ for SRR loaded with $C=0.2 \mathrm{pF}$ and to $2.86 \mathrm{GHz}$ for $C=3.3 \mathrm{pF}$.

We also performed simulations to check the experimental results. Simulations are performed by using commercial software CST MICROWAVE STUDIO, which is a threedimensional (3D) full-wave solver employing the finite integration technique (FIT). ${ }^{14}$ In order to determine the resonance frequencies of the structures under consideration, we have included one layer of the SRR structures along the propagation direction. In the simulation setup, the structures are subjected to an incident plane wave. Open boundary conditions are employed along the propagation direction. Periodic boundary conditions are used along the directions other than the propagation direction. Hence, the structure is assumed to be periodic and infinite along the directions that are perpendicular to the propagation direction. The transmission amplitudes are obtained by using the fields at a distant point from the structures. This point was chosen such that beyond this point the transmission coefficients do not change with increasing distance. Such a choice was made to exclude the near-field effects due to the highly resonant nature of the structures under consideration. The simulation software en-
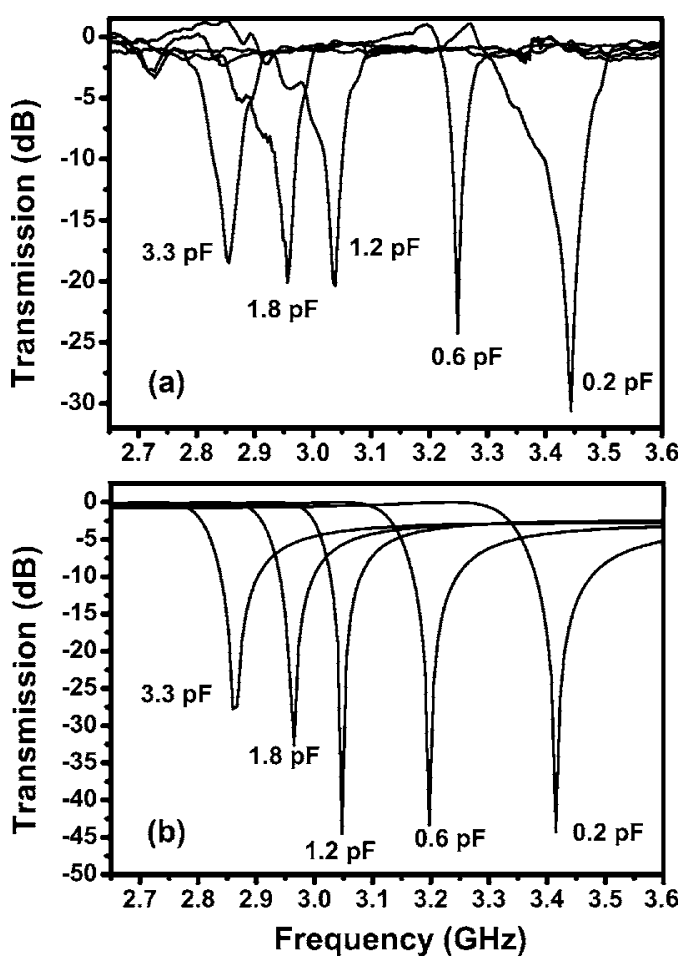

FIG. 3. Transmission coefficients of SRR structures with capacitors loaded at the gap region between inner and outer rings: (a) experimental data, and (b) numerical simulations.

ables us to insert lumped elements into the structure. We inserted capacitors and assigned capacitance values by using this tool. Figure 3(b) shows the simulated transmission spectra, and there is a good agreement between experimental results and numerical simulations.

Secondly, we integrated capacitors at the split region of the outer ring [see Fig. 2(b)]. Measured transmission coefficients of SRRs with different capacitors are provided in Fig. 4(a). Resonance frequency significantly shifts to $2.87 \mathrm{GHz}$ when $C=0.1 \mathrm{pF}$ is mounted to the outer ring's split region. Magnetic resonance frequency can further be reduced down to $0.99 \mathrm{GHz}$ by using a $C=2.2 \mathrm{pF}$ capacitor. The tuning range is higher for a capacitance loaded at the split region than a capacitance loaded at the gap region. Numerical results that are provided in Fig. 4(b) agree quite well with the experimental data. A similar experiment was performed in an earlier study of our group. ${ }^{6}$ We included experimental results with different capacitance values in this letter to make a comparison for the cases where the capacitances are loaded at the inner split and gap regions. We also decreased the magnetic resonance frequency furthermore in this study.

Finally, we loaded capacitances at the split region of the inner ring [see Fig. 2(c)]. Figures 5(a) and 5(b) display measured and calculated transmission coefficients for different capacitance values, respectively. For $C=0.2 \mathrm{pF}$ and $C$ $=1.2 \mathrm{pF}$ the resonances are observed at 3.24 and $1.76 \mathrm{GHz}$, respectively.

The magnetic resonance frequencies of SRRs as a function of capacitances loaded at different capacitive regions are shown in Fig. 6 with corresponding data points. It is clear from Fig. 6 that the highest amount of tuning can be achieved by loading capacitances at the outer split region. 

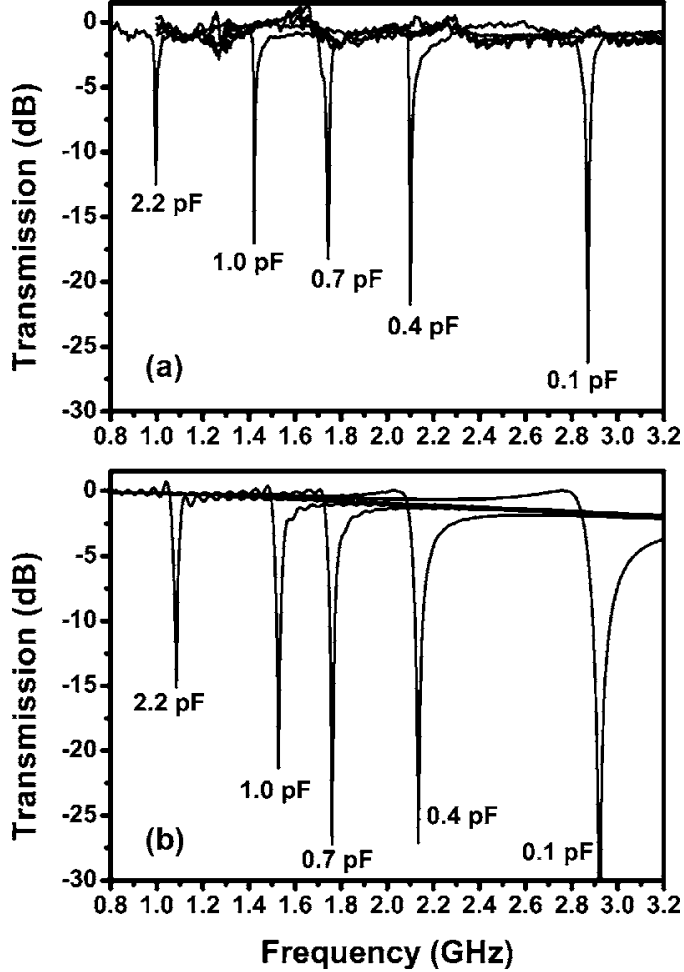

FIG. 4. Transmission coefficients of SRR structures with capacitors loaded at the outer split region: (a) experimental data and (b) numerical simulations.

We also checked the experimental results with the numerical models in the literature. The capacitances due to the splits were ignored and only gap capacitance is taken into account when modeling SRR structure in Ref. 9 and 10.

In general, the split capacitances are relatively small and can be ignored. However, if a capacitor is loaded in the split region, the split capacitance becomes important and affects resonance frequency as observed in our study. In Refs. 11 and 12, the capacitances due to the splits were taken into account; therefore we will use this model to compare our experimental results. The approximate formula for the lowest magnetic resonance frequency is given in Ref. 12 as

$$
\omega_{m}=\sqrt{2 \pi L_{\mathrm{av}}\left[\left(\pi C_{g} / 2\right)+C_{s 1}+C_{s 2}\right]},
$$

where $L_{\mathrm{av}}$ is the average inductance of the two rings, $C_{g}$ is the capacitance due to the gap, and $C_{s 1}$ and $C_{s 2}$ are capacitances due to the outer and inner splits, respectively. By using the formula provided in Ref. 10, we calculated the gap capacitance as $C_{g}=0.14 \mathrm{pF}$. The capacitances due to the splits are calculated to be $C_{s 1}=C_{s 2}=1.2 \mathrm{fF}$. Since the magnetic resonance frequency of our SRR structure is at $\omega_{m}$ $=3.82 \mathrm{GHz}$, by substituting the known parameters in (1) the average inductance is found to be $L_{\mathrm{av}}=76.7 \mathrm{nH}$. The capacitors inserted at the split region is connected in parallel to the split capacitance, therefore we can insert $C_{s 1}=C$ in (1) and plot the corresponding curve for the outer split. Note that $C$ is the capacitance value of the surface mount capacitor. The results obtained from the model are plotted with a blue line in Fig. 6 and the results extremely agree well with experimental data. It is clear from the experimental observations that the effect of the capacitance in the inner split region is
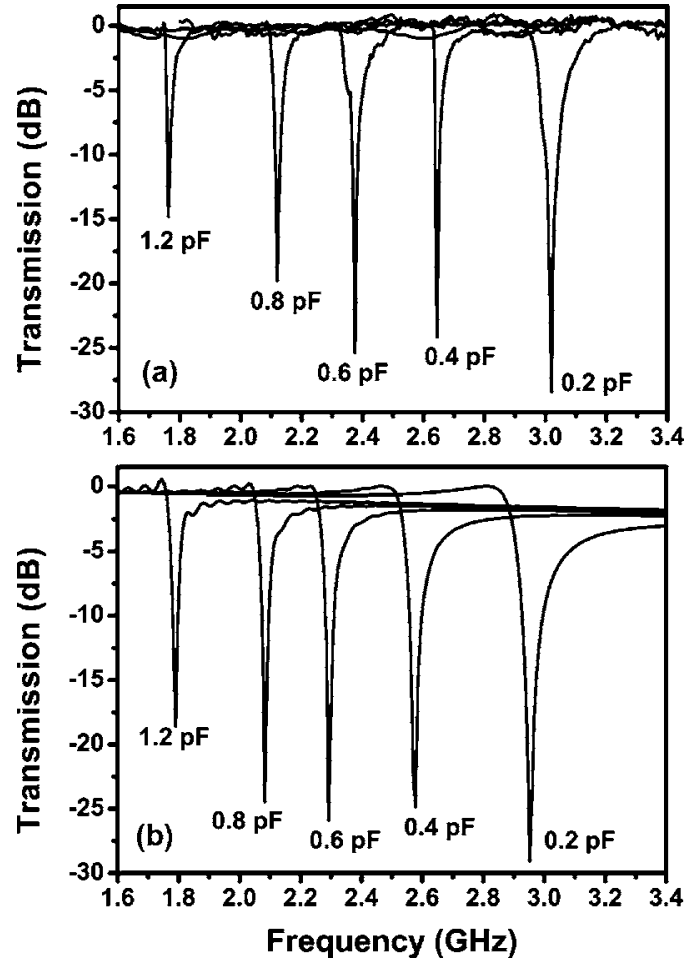

FIG. 5. Transmission coefficients of SRR structures with capacitors loaded at the inner split region: (a) experimental data and (b) numerical simulations.

less than that of the outer split region. Therefore we can introduce a phenomenological factor in the model to obtain the best fit with the experimental data. In (1) if we put $0.4 C_{s 2}$ instead of $C_{s 2}$, the red line in Fig. 6 is obtained. As clearly seen, the match between the model and the experimental results is quite good.

To obtain the results for the capacitor loaded at the gap region, we can use the model by Marques et al. ${ }^{9}$ In the model the rings are divided into two parts as right and left halves. These two halves are connected in series. When we insert the capacitor at the gap region, we connect it in parallel to one half. It is clear that the amount of increase in the gap capacitance will not be as high as in the cases in the outer and inner splits since two halves are connected in series. By using the

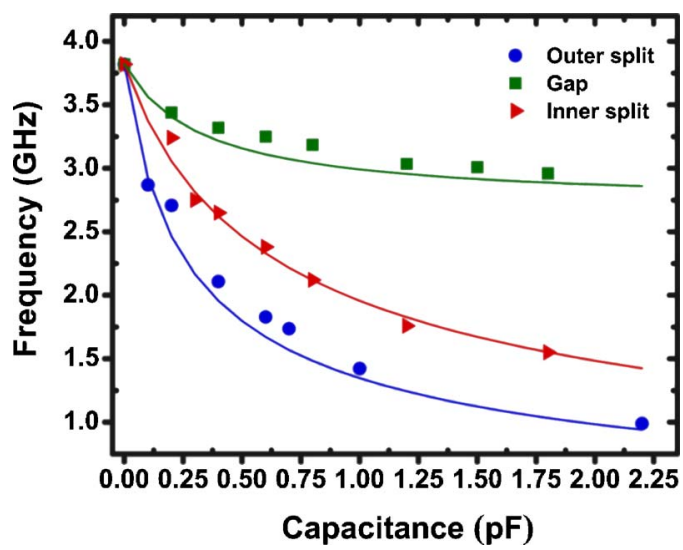

FIG. 6. (Color online) Magnetic resonance frequency of a split ring resonator as a function of loaded capacitances at different capacitive regions. The solid lines are the results obtained from the numerical models. 


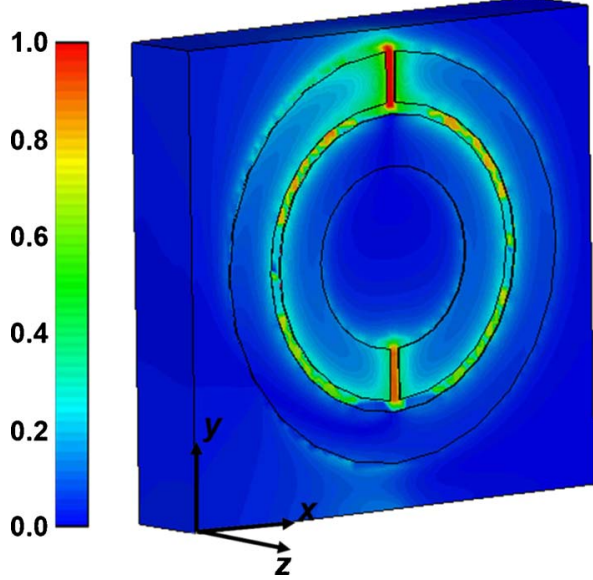

FIG. 7. (Color online) Simulated electric field intensity profile at the magnetic resonance frequency of the split ring resonator.

model proposed in, Ref. 9, we plotted the green curve for the capacitors loaded at the gap region, which also agrees well with the experimental results. The model in Ref. 11 also gives the same result, since it is the special case of the model $^{9}$ with the split capacitances taken into account.

As a comparison, $\omega_{m}$ shifted down to $2.86 \mathrm{GHz}$ with a $3.3 \mathrm{pF}$ capacitor loaded at the gap region. But the same amount of tuning has been achieved with a $0.1 \mathrm{pF}$ capacitor at the split region of the outer ring. To reduce $\omega_{m}$ down to $2.86 \mathrm{GHz}$, a capacitor with capacitance value between 0.2 and $0.4 \mathrm{pF}$ is required to be loaded at the inner ring's split region. Instead of mounting varactors at the gap region, putting them at the split regions should result in higher tunability ranges for SRR structures. ${ }^{13}$ The highest tunability range is achieved with capacitors at the outer ring's split region. Pendry et al. argued that the energy is concentrated at the very small volumes of the SRRs. ${ }^{1}$ Therefore by loading capacitors at small volumes of SRR, such as gaps or splits, one can achieve high EM response from the SRR structures depending on the position of the loading.

We have simulated the electric field intensity at the resonance frequency of SRR by using CST MICROWAVE STUDIO. ${ }^{14}$ An electric field monitor is placed at the resonance frequency and the resulting intensity profile is plotted in Fig. 7. The intensities are normalized with respect to the maximum intensity value. It is clear that the electric field is mostly concentrated at the outer ring's split region. The $E$ field is also localized at the inner split region and gap region, but localization is not as strong as the case in the outer split region. Therefore one should consider using a split region to achieve tunability or nonlinearity from SRR structures instead of the gap region. By loading capacitances, we not only change the magnetic resonance frequency but also change the effective permeability values of SRR. Lee et al. studied the effect of tunable components on the effective parameters of SRR structure. $^{15}$

It is worth mentioning at this point that by loading the capacitor at the split region of the outer ring with a value of $C=2.2 \mathrm{pF}$, we managed to obtain $\omega_{m}$ at $0.99 \mathrm{GHz}$. This is the highest reduction of resonance frequency of a SRR. The diameter of the SRR structure is $7.2 \mathrm{~mm}$ and the free-space wavelength at $0.99 \mathrm{GHz}$ is $303 \mathrm{~mm}$. Therefore magnetic resonance is achieved by using a subwavelength structure with a size of $\lambda / 42$. It is worth mentioning at this point that it is possible to obtain magnetic resonances with lower frequencies by making use of other resonator systems. The size of a capacitively loaded loop is $\lambda / 500,{ }^{16}$ a wide capacitively loaded ring is $\lambda / 320,{ }^{17}$ and a capacitively loaded doublesided spiral is $\lambda / 250,{ }^{18}$ where $\lambda$ corresponds to the wavelength at the magnetic resonance. Another prominent structure is a Swiss roll with a relative diameter of $\lambda / 700 .{ }^{19}$

\section{CONCLUSION}

We used several capacitors with varying values of capacitances to load the capacitive regions of SRR. Experimental and numerical results show that the position of the capacitor affects $\omega_{m}$ considerably. We also validated the results by using the numerical models given in Refs. 9 and 11. The highest reduction of $\omega_{m}$ is achieved when capacitors are loaded at the outer ring's split region. By using SRRs with subwavelength sizes $(\lambda / 42)$, it is possible to obtain magnetic resonance. We believe that our study will shed light on further numerical models of the SRR structures. Capacitorloaded SRRs can be used as tunable metamaterial components, where one can change the magnetic resonance frequency, negative refractive index region, and effective parameters of SRRs.

\section{ACKNOWLEDGMENTS}

This work is supported by the European Union under the projects EU-DALHM, EU-NOE-METAMORPHOSE, EUNOE-PHOREMOST, and TUBITAK under Project Nos. 104E090, 105E066, and 105A005. One of the authors (E. O.) also acknowledges partial support from the Turkish Academy of Sciences. Another author (K. A.) thanks M. D. Caliskan for his help in mounting the capacitors.

${ }^{1}$ J. B. Pendry, A. J. Holden, D. J. Robbins, and W. J. Stewart, IEEE Trans. Microwave Theory Tech. 47, 2075 (1999).

${ }^{2}$ D. R. Smith, W. J. Padilla, D. C. Vier, S. C. Nemat-Nasser, and S. Schultz, Phys. Rev. Lett. 84, 4184 (2000).

${ }^{3}$ K. Aydin, K. Guven, M. Kafesaki, L. Zhang, C. M. Soukoulis, and E. Ozbay, Opt. Lett. 29, 2623 (2004)

${ }^{4}$ K. Aydin, K. Guven, C. M. Soukoulis, and E. Ozbay, Appl. Phys. Lett. 86, 124102 (2005).

${ }^{5}$ K. Aydin, K. Guven, N. Katsarakis, C. M. Soukoulis, and E. Ozbay, Opt. Express 12, 5896 (2004).

${ }^{6}$ K. Aydin, I. Bulu, K. Guven, M. Kafesaki, C. M. Soukoulis, and E. Ozbay, New J. Phys. 7, 168 (2005)

${ }^{7}$ T. Weiland, R. Schuhmann, R. B. Greegor, C. G. Parazzoli, A. M. Vetter, D. R. Smith, D. C. Vier, and S. Schultz, J. Appl. Phys. 90, 5419 (2001).

${ }^{8}$ P. Gay-Balmaz and O. J. F. Martin, J. Appl. Phys. 92, 2929 (2002).

${ }^{9}$ R. Marques, F. Mesa, J. Martel, and F. Medina, IEEE Trans. Antennas Propag. 51, 2572 (2003).

${ }^{10}$ B. Sauviac, C. R. Simovski, and S. Tretyakov, Electromagnetics 24, 317 (2004).

${ }^{11}$ M. Shamonin, E. Shamonina, V. Kalinin, and L. Solymar, Microwave Opt. Technol. Lett. 44, 133 (2005).

${ }^{12}$ A. Radkovskaya, M. Shamonin, C. J. Stevensm, G. Faulkner, D. J. Edwards, E. Shamonina, and L. Solymar, Microwave Opt. Technol. Lett. 46, 473 (2005). 
${ }^{13}$ I. Gil, J. Garcia-Garcia, J. Bonache, F. Martin, M. Sorolla, and R. Marques, Electron. Lett. 40, 1347 (2004).

${ }^{14}$ User Manual, Version 5.0, CST GmbH, Darmstadt, Germany, 2005, http://www.cst. de

${ }^{15}$ S.-W. Lee, Y. Kuga, and A. Ishimaru, Electromagn. Waves 51, 219 (2005).
${ }^{16}$ M. C. K. Wiltshire, E. Shamonina, I. R. Young, and L. Solymar, Electron. Lett. 39, 215 (2003).

${ }^{17}$ O. Sydoruk et al., Phys. Rev. B 73, 224406 (2006).

${ }^{18}$ R. R. A. Syms, I. R. Young, and L. Solymar, J. Phys. D 39, 3945 (2006).

${ }^{19}$ M. C. K. Wiltshire, J. B. Pendry, I. R. Young, D. J. Larkman, D. J. Gilderdale, and J. V. Hajnal, Science 291, 849 (2001). 